\title{
Relación entre el vocabulario comprensivo y la competencia matemática temprana en niños de 5 y 6 años
}

Juan José Yaringaño Limache

\begin{abstract}
Resumen.
El presente estudio tiene como objetivo determinar la relación entre el vocabulario comprensivo y la competencia matemática temprana. Se empleó un tipo de investigación descriptivo correlacional, siendo la muestra de sujetos constituida por 45 niños de una institución educativa estatal. Los resultados indican que existe una correlación significativa entre vocabulario comprensivo y competencia matemática temprana (Rho $=.617)$, además al se observó relación significativa entre vocabulario comprensivo y el componente de capacidad relacional $(\mathrm{Rho}=.661)$ y componente numérico $(\mathrm{Rho}=.441)$ estos resultados permiten concluir que el vocabulario comprensivo asociado a conceptos espaciales, cuantitativos, temporales, cualitativos, de tamaño y forma, se relacionan con la competencia matemática del número.
\end{abstract}

Palabras clave: competencia matemática temprana, vocabulario comprensivo básico, niños de 5 años.

\section{Summary.}

The objective of this study is to determine the relationship between comprehensive vocabulary and early mathematical competence. A type of correlational descriptive research is sought, being the sample of subjects constituted by 45 children of a state educational institution. The results indicate that there is a significant correlation between the comprehensive vocabulary and early mathematical competence (Rho $=.617$ ), in addition to having a rela-tionship between the comprehensive vocabulary and the relational capacity component (Rho $=.661)$ and the numerical component $(\mathrm{Rho}=.441)$. These results allow us to conclude that the comprehensive vocabulary associated with spatial, quantitative, temporal, qualitati-ve concepts of size and form, are related to the mathematical competence of the number.

Key words: Early math competence, Basic comprehension vocabulary, 5-year-old children. 


\section{Introducción}

Al considerar las competencias y habilidades básicas para el aprendizaje de las matemáticas, debemos tener presente que según Piaget (1975) los niños evolucionan a través de una secuencia ordenada de estadios o etapas, en los cuales cada representación que realizan los sujetos del mundo que les rodea es cualitativamente diferente dentro de cada período. En el caso de las habilidades necesarias para la noción de número estas se encuentran asociadas a otras competencias o habilidades, siendo fundamental el nivel de lenguaje comprensivo y expresivo alcanzado. La relevancia de la competencia matemática temprana se centra en que debemos considerarlo un requisito para ser capaz de comenzar el aprendizaje de la matemática, Aunio, Heiskari y Van Luit (2014) observaron que las diferencias en las capacidades iniciales de cálculo entre los niños ya son visibles en la etapa inicial de su vida escolar, específicamente son las diferencias en habilidades relacionales en un contexto numérico y las habilidades de conteo. De este modo el estudio de competencias o habilidades tempranas, permitirían predecir dificultades de aprendizaje de matemáticas.

De acuerdo con García (2015) es fundamental la evaluación del vocabulario comprensivo de los niños pues el dominio de distintos conceptos es necesario para progresar en el aprendizaje escolar. Tapia (2000) señalo la importancia del desarrollo del lenguaje como requisito fundamental para el éxito académico y por ser un medio de comunicación, expresión de desarrollo cognitivo y afectivo, de control y regularización del comportamiento humano. De este modo la capacidad del niño para interactuar y comprender su entorno está basada en el conocimiento del lenguaje. Del mismo modo Belinchon, Riviere e Igoa (1992) señala que en lo que respecta al estudio del lenguaje infantil, la evaluación debe centrarse en el cómo y el qué es lo que comunica el niño, siendo el nivel de complejidad sintáctica y el desarrollo del vocabulario se indicadores importantes para conocer el nivel de desarrollo cognitivos del niño.

Para Van de Rijt et al. (1994), las habilidades matemática tempranas se pueden centrar en áreas específicas como son la comparación, clasificación, correspondencia, seriación, conteo (verbal, estructurado y resultante) y conocimiento general de los números, en niños y niñas de edades entre cuatro y siete años. La competencia matemática se hace necesaria para afrontar las necesidades del entorno, pues se requiere realizar una serie de actividades cognitivas que vinculen las matemáticas a su mundo real, con lo cual adquirirá confianza y pre-paración gradual para enfrentar los nuevos retos de su formación académica. De acuerdo con lo anterior es importante contar con un instrumento de evaluación de las competencias matemáticas básicas, necesidad que plantea como objetivo estimar la validez y confiabilidad de un test que mida las competencias matemáticas tempranas.

Establecer como objetivo de investigación conocer la relación entre el vocabulario comprensivo y el nivel de competencia matemática básica, permite determinar el grado y tipo de relación que se establecen en estas dos variables fundamentales en el desarrollo cognitivo de los niños de 5 y 6 años, siendo estos aspectos requisitos relevantes para los futuros aprendizajes. 


\section{Método}

Tipo y diseño de investigación. La investigación es de tipo cuantitativa y descriptivo correlacional. Se utilizó un diseño no experimental transversal. Describiéndose los puntajes obtenidos en cultura financiera según variables socio demográficas (Hernández, Fernández y Baptista, 2010). También se puede considerar como una investigación psicométrica.

Sujetos. Para la realización de la investigación, se realizará la aplicación de los instrumentos en un grupo de niños de 5 y 6 años de una institución educativa del distrito de Santiago de Surco, la composición de la muestra se detalla en la Tabla 1.

Tabla 1. Descripción de la población según género y edad

\begin{tabular}{|c|c|c|c|}
\hline Hombres & Mujeres & 5 años & 6 años \\
\hline 26 & 20 & 23 & 23 \\
\hline
\end{tabular}

Instrumento. Se utilizarán dos instrumentos, el primero es el Test de Evaluación Matemática Temprana (TEMT) examina dos dimensiones principales de la competencia matemática temprana: aspectos relacionales y aspecto numérico. La dimensión relacional está compuesta por las competencias específicas de Comparación, Clasificación, Correspondencia y Seriación, y en el caso de la dimensión numérica se componen de las competencias específicas de Conteo verbal, Conteo estructurado, Conteo resultante o resultado del conteo y Conocimiento general de los números. El segundo instrumento es el test Magallanes de Vocabulario Comprensivo Básico (TMVCB), que evalúa conceptos espaciales, cuantitativos, temporales, cualitativos, de tamaño y forma.

Análisis estadístico. El análisis estadístico se realizó utilizando medidas de tendencia central (media), estadísticos de dispersión (Desviación Estándar y Varianza), además se realizará los cálculos para obtener los coeficientes de correlación (Rho de Spearman).

\section{Resultados}

A continuación, se presentan los resultados obtenidos para determinar la correlación entre las variables vocabulario comprensivo básico y las competencias matemáticas tempranas.

Los resultados descriptivos de la aplicación de la prueba TMVCB se observan en la Tabla 2, señalan que en vocabulario comprensivo básico los niños de 5 años presentaron una media $=44.82$ y desv est $=4.666$, y los niños de 6 años media $=45.48$ y desv est $=6.563$. Además, se presentan los resultados obtenidos en las dimensiones que lo componen. 
Tabla 2. Estadísticos de tendencia central en vocabulario comprensivo

\begin{tabular}{|l|l|l|l|l|}
\hline \multirow{2}{*}{\multicolumn{1}{c|}{ Dimensiones }} & \multicolumn{1}{c|}{$\mathbf{5}$ años } & \multicolumn{1}{c|}{$\mathbf{N = 2 3}$} & $\mathbf{6}$ años & \multicolumn{1}{c|}{$\mathbf{N = 2 3}$} \\
\cline { 2 - 6 } & \multicolumn{1}{|c|}{$\mathbf{M}$} & \multicolumn{1}{c|}{ DS } & \multicolumn{1}{c|}{$\mathbf{M}$} & \multicolumn{1}{c|}{ DS } \\
\hline Espaciales & 14.86 & 2.210 & 15.26 & 1.888 \\
\hline Cuantitativos & 6.41 & 0.854 & 6.35 & 1.335 \\
\hline Temporales & 3.23 & 0.813 & 3.43 & 0.843 \\
\hline Cualitativos & 14.14 & 1.246 & 14.43 & 2.150 \\
\hline Tamaño y forma & 6.18 & 0.795 & 6.00 & 1.279 \\
\hline Vocabulario comprensivo (Total) & 44.82 & 4.666 & 45.48 & 6.563 \\
\hline
\end{tabular}

Los resultados descriptivos de la aplicación de la prueba TEMT se observan en la Tabla 3, señalan que en competencia matemática básica los niños de 5 años, presentaron una media $=23.73$ y desv est $=7.032$ y los niños de 6 años media $=26.35$ y desv est $=7.499$. Además, se presentan los resultados de acuerdo con las competencias Relacional y Numeracional así como sus componentes.

Tabla 3. Estadísticos de tendencia central en competencia matemática temprana

\begin{tabular}{|c|c|c|c|c|}
\hline & 5 años & $\mathrm{N}=\mathbf{2 3}$ & 6 años & $\mathrm{N}=\mathbf{2 3}$ \\
\hline & M & DS & M & DS \\
\hline Comparación & 4.36 & 1.177 & 4.57 & .945 \\
\hline Clasificación & 3.64 & 1.255 & 3.74 & 1.137 \\
\hline Correspondencia & 3.41 & 1.297 & 3.57 & 1.343 \\
\hline Seriación & 2.41 & 1.368 & 3.35 & 1.071 \\
\hline Competencia Relacional & 13.82 & 4.113 & 15.22 & 3.357 \\
\hline Conteo verbal & 2.77 & 1.541 & 2.70 & 1.521 \\
\hline Conteo estructural & 2.55 & 1.262 & 3.00 & 1.206 \\
\hline Conteo resultante & 1.68 & 1.171 & 2.30 & 1.608 \\
\hline Conocimiento general de los números & 2.91 & 1.571 & 3.13 & 1.456 \\
\hline Competencia Numeracional & 9.91 & 4.396 & 11.13 & 4.780 \\
\hline Total & 23.73 & 7.032 & 26.35 & 7.499 \\
\hline
\end{tabular}

La tabla 4, plantea si existe relación entre el vocabulario comprensivo básico y competencia matemática temprana y sus dimensiones en niños de 5 y 6 años. En esta tabla se recoge la información referida al coeficiente rho de Spearman. En esta tabla aparecen el valor del coeficiente de correlación, el nivel crítico asociado a cada coeficiente (Sig.) y el número de casos. Basándonos en los niveles críticos de la tabla 4, se puede afirmar que las variables el vocabulario comprensivo básico y la competencia matemática temprana, si correlacionan significativamente (Rho=0,000). 
Tabla 17. Coeficientes rho de Spearman, para determinar la correlación entre comprensión lectora y atención

\begin{tabular}{|l|c|c|}
\hline \multirow{2}{*}{} & \multicolumn{2}{|c|}{ Vocabulario comprensivo básico } \\
\cline { 2 - 3 } & Rho de Spearman & Sig. (bilateral) \\
\hline Competencia Relacional &, $661^{\star \star}$ &, 000 \\
\hline Competencia Numérica &, $441^{\star \star}$ &, 000 \\
\hline Competencia matemática temprana &, $617^{\star \star}$ &, 000 \\
\hline
\end{tabular}

**. La correlación es significativa en el nivel 0,01 (bilateral).

Decisión. De acuerdo con el resultado de la tabla 4, se observa relación significativa entre las puntuaciones de vocabulario comprensivo básico y la competencia matemática temprana, asimismo existe relación significativa entre el vocabulario comprensivo y las competencias relacional y numérica.

\section{Discusión}

De acuerdo con los resultados obtenidos se puede afirmar que el vocabulario comprensivo presenta una relación positiva y de nivel medio con la competencia matemática temprana esto indicaría que el conocimiento de conceptos espaciales, cuantitativos, temporales, cualitativos, de tamaño y forma, está asociada al desarrollo de las competencias matemáticas tempranas de ccomparación, Clasificación, Correspondencia y Seriación. Además, el vocabulario comprensivo se relaciona de marea positiva de nivel medio bajo con las competencias numéricas de Conteo verbal, Conteo estructurado, Conteo resultante o resultado del conteo y Conocimiento general de los números.

El desarrollo del vocabulario comprensivo en el niño es un requisito fundamental para el desempeño escolar académico, a partir de un vocabulario comprensivo adecuado el niño puede comunicar sus experiencias, expresión sus ideas y el modo en que pueden comprender la realidad, esto le permitirá asimilar los conceptos numéricos que le permitan comprender el mundo a partir del uso del conteo y el uso del número. De este modo la capacidad del niño para interactuar y comprender su entorno está basada en el conocimiento del lenguaje. 


\section{REFERENCIAS}

Boehm, A. (2012). Test Boehm de conceptos básicos-3 Preescolar (Boehm-3 Preescolar)- Madrid: TEA.

Belinchón, M., Riviére, A. e lgoa, J. (1992). Psicología del Lenguaje. Investigación y Teoría. Madrid: Trotta.

Cerda, G., Pérez, C., Moreno, C., Núñez, K., Quezada, E., Rebolledo, J. y Sáez, S. (2012). Adaptación de la versión española del Test de Evaluación Matemática Temprana de Utrecht en Chile. Estudios Pedagógicos, 38(1), 235-253.

Garcia, E. (2015). Test Magallanes de Vocabulario Comprensivo Basico (TMVCB). Madrid: AlborCohs.

Hernández, R., Fernández, C. y Baptista, M. (2010). Metodología de la investigación. $5^{a}$ ed. México D.F.: McGraw-Hill Interamericana.

Navarro, J., Aguilar, M., Alcalde, C., Marchena, E., Ruiz, G., Menacho, I. y Sedeño, M. (2011). Test de Evaluación Matemática Temprana (TEMT). Madrid: EOS

Piaget, J. \& Inhelder, B. (1997) Psicología del niño. 14a ed. España. Morata.

Piaget, J. \& Inhelder, B. (1975). Génesis de las Estructuras Lógicas Elementales. Clasificaciones y Seriaciones. Guadalupe, Buenos Aires-

Sánchez, H. \& Reyes, C. (2002). Metodología y diseños en la investigación científica. Lima: Universitaria.

Tapia, V. (2000). Niveles de desarrollo del lenguaje comprensivo en niños de segundo a sexto grado de primaria. Revista de Investigación en Psicología, 3(1), 115-125.

Van De Rijt, B., Van Luit, J. Y Pennings, A. (1999). The construction of the Utrech Early Mathematical Competence Scales. Educational and Psychological Measurement, 59 (2), 289-309. Recuperado de http://www.scielo.cl/pdf/estped/v38n1/art14.pdf 